\title{
LOCALIZING GAUGE THEORIES
}

\author{
Nikita A. NEKRASOV
}

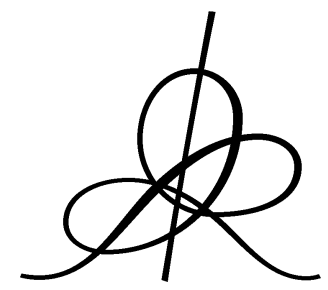

Institut des Hautes Études Scientifiques

35 , route de Chartres

91440 - Bures-sur-Yvette (France)

Décembre 2003

$\mathrm{IHES} / \mathrm{P} / 03 / 66$ 


\title{
LOCALIZING GAUGE THEORIES
}

\author{
NIKITA A. NEKRASOV* \\ Institut des Hautes Etudes Scientifiques \\ 35 route de Chartres, \\ 91440 Bures-sur-Yvette, FRANCE \\ E-mail: nikita@ihes.fr
}

\begin{abstract}
We study $\mathcal{N}=2$ supersymmetric gauge theories on toric four dimensional manifolds. We recall the notion of $\Omega$-background and calculate the partition function of gauge theory in this background. As a result, we get generalizations of the formulae of the author for the theories on $\mathbb{R}^{4}$ and Nakajima-Yoshioka for the theories on $\mathbb{R}^{4}$ blown up at one point. In the case of compact toric space our results give an alternative derivation of Donaldson invariants, and generalize the results of Göttche-Zagier.
\end{abstract}

\section{Introduction}

In the past years some progress has been achieved in the quantum field theory calculations of the low-energy effective actions including non-perturbative effects. $\mathcal{N}=2$ supersymmetric Yang-Mills theory has simple perturbation theory and non-trivial instanton dynamics. By utilizing supersymmetry one is able to calculate the instanton corrections to the low-energy effective action [57]

\subsection{Generalized Scherk-Schwarz construction}

The idea is to put the theory in a nontrivial geometric background, which we presently describe. Consider any Lorentz-invariant field theory in $d$ dimensions. Suppose the theory can be obtained by Kaluza-Klein reduction from some theory in $d+1$ dimensions. In addition, suppose the theory in $d+1$ dimensions had a global symmetry group $H$. Now compactify the $d+1$ dimensional theory on a circle $\mathbb{S}^{1}$ of circumference $r$, with a twist, so that in going around the circle, the space-time $\mathbb{R}^{d}$ experiences a Lorentz rotation, by an element $\exp (r \Omega)$, and in addition a Wilson line in the group $H, \exp (r \mathbb{A})$ is turned on. The resulting theory can be now considered in the $r \rightarrow 0$

\footnotetext{
* On leave of absence from Institute for Theoretical and Experimental Physics
} 
limit, where for finite $\Omega, \mathbb{A}$ we find extra couplings in the $d$-dimensional Lagrangian. This is the background we shall extensively use. Specifically we shall be mostly interested in the four dimensional $\mathcal{N}=2$ theories. They all can be viewed as dimensional reductions of $\mathcal{N}=1$ susy gauge theories from six or five dimensions. The global symmetry group $H$ in six dimensions is $S U(2)$ (R-symmetry).

These considerations lead to powerful results concerning exact nonperturbative calculations in the supersymmetric gauge theories. In particular, one arrives (Ref. [57]) at the technique of deriving effective prepotentials of the $\mathcal{N}=2$ susy gauge theories with the gauge groups $U\left(N_{1}\right) \times \ldots \times U\left(N_{k}\right)$ (based on Refs. [41-43, 49,58], see also Refs. [11]). Previously, the effective low-energy action and the corresponding prepotential $\mathcal{F}^{S W}$ was determined using the constraints of holomorphy and electro-magnetic duality in Refs. [35, 65, 66].

\subsection{Higher Casimirs in gauge theory}

One can also get the correlation functions of $\mathcal{N}=2$ chiral operators. This is equivalent to solving for the effective prepotential of the $\mathcal{N}=2$ theory whose microscopic prepotential (see Ref. [65] for introduction in $\mathcal{N}=2$ susy) is given by:

$$
\mathcal{F}^{U V}=\tau_{0} \operatorname{Tr} \Phi^{2}+\sum_{\vec{n}} \tau_{\vec{n}} \prod_{J=1}^{\infty} \frac{1}{n_{J} !}\left(\frac{1}{J} \operatorname{Tr} \Phi^{J}\right)^{n_{J}}
$$

where $\vec{n}=\left(n_{1}, n_{2}, \ldots\right)$ label all possible gauge-invariant polynomials in the adjoint Higgs field $\Phi$ (note that $\tau_{0,1,0, \ldots}$ shifts $\tau_{0}$ ).

Let $\vec{\rho}=(1,2,3, \ldots),|\vec{n}|=\sum_{J} n_{J}$, and $\vec{n} \cdot \vec{\rho}=\sum_{J} J n_{J}$.

In order for the theory defined by Eq. (1) not to run into strong coupling singularity, i.e. to avoid vanishing of the second derivatives of prepotential at large (quasiclassical) values of the Higgs field

$$
\langle\Phi\rangle_{a} \sim a \gg \Lambda \sim e^{2 \pi i \tau_{0}}
$$

the couplings $\tau_{\vec{n}}$ should be treated formally. One could also worry about the nonrenormalizabilty of the perturbation (1). This is actually not so, provided the conjugate prepotential $\overline{\mathcal{F}}$ is kept classical $\bar{\tau}_{0} \operatorname{Tr} \bar{\Phi}^{2}$. The action is no longer real, however, the effective dimensions of the fields $\Phi$ and $\bar{\Phi}$ become 0 and 2, thereby justifying an infinite number of terms in Eq. (1).

We should note that there are relations between the deformations generated by derivatives w.r.t. $\tau_{\vec{n}}$, which originate in the fact that there are polynomial relations between the single-trace operators $\operatorname{Tr} \Phi^{J}$ for $J>N$ 
and the multiple-trace operators. When instantons are included these classical relations are modified. It seems convenient to keep all $\tau_{\vec{n}}$ as independent couplings. The classical prepotential then obeys additional constraints: the $N$-independent non-linear ones:

$$
\frac{\partial \mathcal{F}^{U V}}{\partial \tau_{\vec{n}}}=\frac{\partial \mathcal{F}^{U V}}{\partial \tau_{\vec{n}_{1}}} \ldots \frac{\partial \mathcal{F}^{U V}}{\partial \tau_{\vec{n}_{k}}}, \quad \vec{n}=\vec{n}_{1}+\ldots \vec{n}_{k}
$$

and the $N$-dependent linear ones:

$$
\sum_{\vec{n}: \vec{n} \cdot \vec{\rho}=N+k}(-1)^{|\vec{n}|} \frac{\partial}{\partial \tau_{\vec{n}}} \mathcal{F}^{U V}=0, \quad k>0
$$

The quantum effective prepotential obeys instanton corrected constraints [42], which we implicitly determine in this paper.

\subsection{Contact terms}

The constraints of holomorphy and electro-magnetic duality are powerful enough to determine the effective low-energy prepotential $\mathcal{F}^{I R}$ (see Ref. [42]), up to a diffeomorphism of the couplings $\tau_{\vec{n}}$, i.e. up to contact terms. In order to fix the precise mapping between the microscopic couplings (which we also call "times", in accordance with the terminology adopted in integrable systems) and the macroscopic ones, one needs more refined methods (see Ref. [43] for the discussion of the contact terms and their relation to the topology of the compactifications of the moduli spaces). As we shall explain in this paper, the direct instanton calculus is powerful enough to solve for $\mathcal{F}^{I R}$ :

$$
\mathcal{F}^{I R}\left(a, \tau_{\vec{n}}\right)=\mathcal{F}^{S W}\left(a ; \tau_{0}\right)+\sum_{\vec{n}} \tau_{\vec{n}} \mathcal{O}_{\vec{n}}(a)+\sum_{\vec{n}, \vec{m}} \tau_{\vec{n}} \tau_{\vec{m}} \mathcal{O}_{\vec{n} \vec{m}}(a)+\ldots
$$

where

$$
\mathcal{O}_{\vec{n}}(a)=\left\langle\prod_{J=1}^{\infty} \frac{1}{n_{J} !}\left(\frac{1}{J} \operatorname{Tr} \Phi^{J}\right)^{n_{J}}\right\rangle_{a}
$$

while $\mathcal{O}_{\vec{n} \vec{m}}$ are the expectation values of the contact terms between $\mathcal{O}_{\vec{n}}$ and $\mathcal{O}_{\vec{m}}[24,42,50]$.

\subsection{Looking for holomorphic anomaly}

The partition function of the gauge theory in $\Omega$-background is analytic in the vev of the Higgs field. However the expansion in the parameters $\epsilon_{1}, \epsilon_{2}$ 
in the limit $\epsilon \rightarrow 0$ leads to holomorphic functions in $a, \mathcal{F}_{0}(a ; \Lambda), \mathcal{F}_{1}(a ; \Lambda)$ etc which are actually not single-valued. They can be made single valued at the expense of adding some non-holomorphic terms (the example of the second Eisenstein series $E_{2}$ is helpful here). The precise expression of these non-holomorphic terms is not known. We are proposing an approach below which should fix them, at least partly.

Another, more profound, reason to expect them is to recall the GromovWitten interpretation of the $\mathcal{N}=2$ partition functions. At least in the case $\epsilon_{1}=-\epsilon_{2}$ the expansion coefficients $\mathcal{F}_{g}(a ; \Lambda)$ are (the limits of) the generating functions of the genus $g$ Gromov-Witten invariants of certain (local) Calabi-Yau manifolds, see Ref. [70]. These are well-known to be the large $\bar{a}$ limits of non-holomorphic automorphic forms on the moduli spaces of Kahler structures of these Calabi-Yaus, see Refs. [4,9].

\section{2. $\mathcal{N}=2$ THEORY}

\subsection{Gauge theory realizations}

We start our exposition with the case of pure $\mathcal{N}=2$ supersymmetric YangMills theory with the gauge group $U(N)$ and its maximal torus $\mathbf{T}=U(1)^{N}$. The field content of the theory is given by the vector multiplet $\Phi$, whose components are: the complex scalar $\Phi$, two gluions $\lambda_{\alpha}^{i}, i=1,2 ; \alpha=1,2$ their conjugates $\bar{\lambda}_{\dot{\alpha} i}$, and the gauge field $A_{\mu}$ - all fields in the adjoint representation of $U(N)$. The action is given by the integral over the superspace:

$$
S \propto \int d^{4} x\left(\int d^{4} \theta \mathcal{F}(\Phi)+\int d^{4} \bar{\theta} \overline{\mathcal{F}}(\bar{\Phi})\right)
$$

where $\theta_{\alpha}^{i}, \alpha=1,2 ; i=1,2$ are the chiral Grassmann coordinates on the superspace, $\Phi=\Phi+\theta \lambda+\theta \theta F^{-}+\ldots$ is the $\mathcal{N}=2$ vector superfield, and $\mathcal{F}$ is the prepotential (locally, a holomorphic gauge invariant function of $\Phi$ ). Classical supersymmetric Yang-Mills theory has

$$
\mathcal{F}(\Phi)=\tau_{0} \operatorname{Tr} \Phi^{2}
$$

where $\tau_{0}$ is a complex constant, whose real and imaginary parts give the theta angle and the inverse square of the gauge coupling respectively:

$$
\tau_{0}=\frac{\vartheta_{0}}{2 \pi}+\frac{4 \pi i}{g_{0}^{2}}
$$

the subscript 0 reminds us that these are bare quantities, defined at some high energy scale $\mu_{U V}$. It is well-known that $\mathcal{N}=2$ gauge theory has a moduli space of vacua, characterized by the expectation value of the complex 
scalar $\Phi$ in the adjoint representation. In the vacuum $[\Phi, \bar{\Phi}]=0$, due to the potential term $\operatorname{Tr}[\Phi, \bar{\Phi}]^{2}$ in the action of the theory. Thus, one can gauge rotate $\Phi$ to the Cartan subalgebra of $\mathfrak{g}:\langle\Phi\rangle=a \in \mathbf{t}=\operatorname{Lie}(\mathbf{T})$. We are studying the gauge theory on Euclidean space $\mathbb{R}^{4}$, and impose the boundary condition $\Phi(x) \rightarrow a$, for $x \rightarrow \infty$. It is also convenient to accompany the fixing of the asymptotics of the Higgs field by the fixing the allowed gauge transformations to approach unity at infinity.

The $\mathcal{N}=2$ gauge theory in four dimensions is a dimensional reduction of the $\mathcal{N}=1$ five dimensional theory. The latter theory needs an ultraviolet completion to be well-defined. However, some features of its low-energy behavior are robust [74].

In particular, the effective gauge coupling runs because of the one-loop vacuum polarization by the BPS particles. These particles are W-bosons (for nonabelian theory), four dimensional instantons, viewed as solitons in five dimensional theory, and the bound states thereof.

To calculate the effective couplings we need to know the multiplicities, the masses, the charges, and the spins of the BPS particles present in the spectrum of the theory $[25,58]$. This can be done, in principle, by careful quantization of the moduli space of collective coordinates of the soliton solutions (which are four dimensional gauge instantons). Now suppose the theory is compactified on a circle. Then the one-loop effect of a given particle consists of a bulk term, present in the five dimensional theory, and a new finite-size effect, having to do with the loops wrapping the circle in spacetime [58]. If in addition the noncompact part of the space-time in going around the circle is rotated then the loops wrapping the circle would have to be localized near the origin in the space-time. This localization is at the core of the method we are employing. Its mathematical implementation is discussed in the next section. Physically, the multiplicities of the BPS states are accounted for by the supersymmetric character-valued index [25]:

$$
\sum_{\text {solitons }} \operatorname{Tr}_{\mathcal{H}}(-)^{F} e^{-r \mathbb{P}_{5}} e^{r \Omega \cdot \mathbb{M}} e^{r \mathbb{A} \cdot \mathbb{I}}
$$

where $\mathbb{P}_{5}$ is the momentum in the fifth direction, $\mathbb{M}$ is the generator of the Lorentz rotations, $\mathbb{I}$ is the generator of the R-symmetry rotations, and $r$ is the circumference of the fifth circle. Under certain conditions on $\Omega$ and $\mathbb{A}$ this trace has some supersymmetry which allows to evaluate it. In the process one gets some integrals over the instanton collective coordinates, as in Refs. $[13,49,67,68]$. As in Ref. [49], these integrals are exactly calculable, thanks to the equivariant localization, described in appendix. 
Another point of view on our method is that by appropriately deforming the theory (in a controllable way) we achieve that the path integral has isolated saddle points, and thanks to the supersymmetry is exactly given by the WKB approximation. The final answer is then the sum over these critical points of the ratio of bosonic and fermionic determinants. This sum is shown to be equal to the partition function of an auxilliary statistical model, desribing the random growth of the Young diagrams. We describe this model in detail in the section 2.7.

We now conclude our discussion of the reduction of the five dimensional theory down to four dimensions. Actually, we can be more general, and discuss the reduction from six dimensions.

Consider lifting the $\mathcal{N}=2$ four dimensional theory to $\mathcal{N}=(1,0)$ six dimensional theory, and then compactifying on a two-torus with the twisted boundary conditions (along both $A$ and $B$ cycles), such that as we go around a non-contractible loop $\ell \sim n A+m B$, the space-time and the fields of the gauge theory charged under the R-symmetry group $S U(2)_{I}$ are rotated by the element $\left(e^{i\left(n a_{1}+m b_{1}\right) \sigma_{3}}, e^{i\left(n a_{2}+m b_{2}\right) \sigma_{3}}, e^{i\left(n a_{2}+m b_{2}\right) \sigma_{3}}\right) \in S U(2)_{L} \times S U(2)_{R} \times$ $S U(2)_{I}=S \sin (4) \times S U(2)_{I}$. In other words, we compactify the six dimensional $\mathcal{N}=1$ susy gauge theory on the manifold with the topology $\mathbb{T}^{2} \times \mathbb{R}^{4}$ with the metric and the R-symmetry gauge field Wilson line:

$$
\begin{gathered}
d s^{2}=r^{2} d z d \bar{z}+\left(d x^{\mu}+\Omega_{\nu}^{\mu} x^{\nu} d z+\bar{\Omega}_{\nu}^{\mu} x^{\nu} d \bar{z}\right)^{2} \\
\mathbb{A}^{a}=\left(\Omega^{\mu \nu} d z+\bar{\Omega}^{\mu \nu} d \bar{z}\right) \eta_{\mu \nu}^{a}, \mu=1,2,3,4, a=1,2,3
\end{gathered}
$$

where $\eta$ is the anti-self-dual 't Hooft symbol. It is convenient to combine $a_{1,2}$ and $b_{1,2}$ into two complex parameters $\epsilon_{1,2}$ :

$$
\epsilon_{1}-\epsilon_{2}=2\left(a_{1}+i b_{1}\right), \quad \epsilon_{1}+\epsilon_{2}=2\left(a_{2}+i b_{2}\right)
$$

The antisymmetric matrices $\Omega, \bar{\Omega}$ are given by:

$$
\Omega=\frac{\epsilon_{1}+\epsilon_{2}}{2} J_{3}^{L}+\frac{\epsilon_{1}-\epsilon_{2}}{2} J_{3}^{R}, \quad \bar{\Omega}=\frac{\bar{\epsilon}_{1}+\bar{\epsilon}_{2}}{2} J_{3}^{L}+\frac{\bar{\epsilon}_{1}-\bar{\epsilon}_{2}}{2} J_{3}^{R}
$$

where we have used the decomposition $S U(2)_{L} \times S U(2)_{R}$ of the rotation group $\operatorname{Spin}(4)$. Clearly, $[\Omega, \bar{\Omega}]=0$. In the limit $r \rightarrow 0$ we get four dimensional gauge theory. We could also take the limit to the five dimensional theory, by considering the degenerate torus $\mathbb{T}^{2}$. We note in passing that the complex structure of the torus $\mathbb{T}^{2}$ could be kept finite. The resulting four dimensional theory (for gauge group $S U(2)$ ) is related to the theory of the so-called E-strings $[23,51]$. The instanton contributions to the correlation functions of the chiral operators in this theory are related to the elliptic 
genera of the instanton moduli space [8] and could be summed up, giving rise to the Seiberg-Witten curves for these theories. However, in this paper we shall neither discuss elliptic, nor trigonometric limits, even though they lead to interesting integrable systems [73].

The action of the four dimensional theory in the limit $r \rightarrow 0$ is not that of the pure supersymmetric Yang-Mills theory on $\mathbb{R}^{4}$. Rather, it is a deformation of the latter by the $\Omega, \bar{\Omega}$-dependent terms. We shall write down here only the terms with bosonic fields (for simplicity, we have set $\vartheta_{0}=0$ ):

$S(\Omega)^{b o s}=-\frac{1}{2 g_{0}^{2}} \operatorname{Tr}\left(\frac{1}{2} F_{\mu \nu}^{2}+\left(D_{\mu} \Phi-\Omega_{\lambda}^{\nu} x^{\lambda} F_{\mu \nu}\right)\left(D_{\mu} \bar{\Phi}-\bar{\Omega}_{\lambda}^{\nu} x^{\lambda} F_{\mu \nu}\right)+[\Phi, \bar{\Phi}]^{2}\right)$

We shall call the theory (13) an $\mathcal{N}=2$ theory in the $\Omega$-background. It is amusing that this deformation can be indeed described as a superspacedependent bare coupling $\tau_{0}$ :

$$
\tau_{0}\left(x, \theta ; \mu_{U V}\right)=\tau_{0}\left(\mu_{U V}\right)+\bar{\Omega}^{-} \theta \theta+\Omega_{\mu \nu} \bar{\Omega}_{\mu \lambda} x^{\nu} x^{\lambda}
$$

We are going to study the correlation functions of chiral observables. These observables are gauge invariant holomorphic functions of the superfield $\Phi$. Viewed as a function on the superspace, every such observable $\mathcal{O}$ can be decomposed:

$$
\mathcal{O}[\Phi(x, \theta)]=\mathcal{O}^{(0)}+\mathcal{O}^{(1)} \theta+\ldots+\mathcal{O}^{(4)} \theta^{4}
$$

The component $\mathcal{O}^{(4)}$ can be used to deform the action of the theory, this deformation is equivalent to the addition of $\mathcal{O}$ to the bare prepotential.

The nice property of the chiral observables is the independence of their correlation functions of the anti-chiral deformations of the theory, in particular of $\bar{\tau}_{0}{ }^{\mathrm{a}}$. We can, therefore, consider the limit $\bar{\tau}_{0} \rightarrow \infty$. In this limit the term:

$$
\bar{\tau}_{0}\left\|F^{+}\right\|^{2}
$$

in the action localizes the path integral onto the instanton configurations. In addition, the $\Omega$-background further localizes the measure on the instantons, invariant under rotations. Finally, the vev of the Higgs field shrinks these instantons to the points, thus eliminating all integrations, reducing them to the single sum over the point-like invariant instantons.

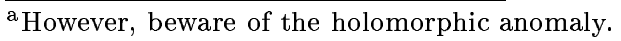


Now we want to pause to discuss other physical realizations of our $\mathcal{N}=2$ theories.

\subsection{String theory realizations}

The $\mathcal{N}=2$ theory can arise as a low energy limit of the theory on a stack of D-branes in type II gauge theory. A stack of $N$ parallel D3 branes in IIB theory in flat $\mathbb{R}^{1,9}$ carries $\mathcal{N}=4$ supersymmetric Yang-Mills theory [81]. A stack of parallel D4 branes in IIA theory in flat $\mathbb{R}^{1,9}$ carries $\mathcal{N}=2$ supersymmetric Yang-Mills theory in five dimensions. Upon compactification on a circle the latter theory reduces to the former in the limit of zero radius.

Now consider the stack of $N$ D4 branes in the geometry $\mathbb{S}^{1} \times \mathbb{R}^{1,8}$ with the metric:

$$
d s^{2}=d x^{\mu} d x^{\mu}+r^{2} d \varphi^{2}+d v^{2}+\left|d Z_{1}+m r Z_{1} d \varphi\right|^{2}+\left|d Z_{2}-m r Z_{2} d \varphi\right|^{2}
$$

Here $x^{\mu}$ denote the coordinates on the Minkowski space $\mathbb{R}^{1,3}, \varphi$ is the periodic coordinate on the circle of circumference $r, v$ is a real transverse direction, $Z_{1}$ and $Z_{2}$ are the holomorphic coordinates on the remaining $\mathbb{C}^{2}$. The worldvolume of the branes is $\mathbb{S}^{1} \times \mathbb{R}^{1,3}$, which is located at $Z_{1}=Z_{2}=0$, and $v=v_{l}, l=1, \ldots, N$. Together with the Wilson loop eigenvalues $e^{i \sigma_{1}}, \ldots, e^{i \sigma_{N}}$ around $\mathbb{S}^{1} v_{l}$ 's form $N$ complex moduli $w_{1}, \ldots, w_{N}$, parameterizing the moduli space of vacua. In the limit $r \rightarrow 0$ the $N$ complex moduli loose periodicity.

It is easy to check that the worldvolume theory has $\mathcal{N}=2$ susy, with the massive hypermultiplet in the adjoint representation (of mass $m$ ). This realization is T-dual to the standard realization with the NS5 branes, as in Ref. [82]. Note that the background (10) is similar to (16). However, the D-branes are differently located, the fact which leads to very interesting geometries upon T-dualities and lifts to M-theory, providing (hopefully) another useful insight.

However, in our story we want to analyze the pure $\mathcal{N}=2$ supersymmetric Yang-Mills theory. This can be achieved by taking $m \rightarrow \infty$ limit, at the same time taking the weak string coupling limit. The resulting brane configuration can be described using two parallel NS5 branes and N D4 brane suspended between them, as in Ref. [82], or, alternatively, as a stack of $N$ D3 (fractional) branes stuck at the $\mathbb{C}^{2} / \mathbb{Z}_{2}$ singularity, as in Ref. [17]. In fact the precise form of the singularity is irrelevant, as long as it corresponds to a discrete subgroup of $S U(2)$, and all the fractional branes are of the same type. The relation between these two pictures is through the T-duality of the resolved $\mathbb{C}^{2} / \mathbb{Z}_{2}$ singularity. The fractional D3 branes blow up into D5 branes wrapping a 
non-contractible two-sphere. The resolved space $T^{*} \mathbb{C P}^{1}$ has a $U(1)$ isometry, with two fixed points (the North and South poles of the non-contractible twosphere). Upon T-duality these turn into two NS5 branes. The D5 branes dualize to D4 branes suspended between NS5's.

The instanton effects in this theory are due to the fractional $\mathrm{D}(-1)$ instantons, which bind to the fractional D3 branes, in the IIB description. The "worldvolume" theory on these $\mathrm{D}(-1)$ instantons is the supersymmetric matrix integral, which we describe with the help of ADHM construction below. In the IIA picture the instanton effects are due to Euclidean D0 branes, which "propagate" between two NS5 branes.

The IIB picture with the fractional branes corresponds to the metric (before $\Omega$ is turned on):

$$
d s^{2}=d x^{\mu} d x^{\mu}+d w d \bar{w}+d s_{\mathbb{C}^{2} / \mathbb{Z}_{2}}^{2}
$$

The singularity $\mathbb{C}^{2} / \mathbb{Z}_{2}$ has five moduli in IIB string theory: three parameters of the geometric resolution of the singularity, and the fluxes of the NSNS and RR 2-forms through the two-cycle which appears after blowup. The latter are responsible for the gauge couplings on the fractional D3 branes, as in Ref. [38]:

$$
\tau_{0}=\int_{\mathbb{S}^{2}} B_{R R}+\tau_{I I B} \int_{\mathbb{S}^{2}} B_{N S N S}
$$

Our conjecture is that turning on the higher Casimirs, (and gravitational descendants on the dual closed string side) corresponds to a "holomorphic wave", where $\tau_{0}$ holomorphically depend on $w$. From Ref. [72] this is known to be a solution of IIB supergravity.

We shall return to the fractional brane picture later on. Right now let us mention another stringy effect. By turning on the constant NSNS B-field along the worldvolume of the D3-branes we deform the super-Yang-Mills on $\mathbb{R}^{4}$ to the super-Yang-Mills on the noncommutative $\mathbb{R}_{\Theta}^{4}$, see Refs. [14,15,75]. On the worldvolume of the $\mathrm{D}(-1)$ instantons the noncommutativity acts as a Fayet-Illiopoulos term, deforming the ADHM equations, as in Refs. [2,3,59], and resolving the singularities of the instanton moduli space, as in Ref. [52]. We shall use this deformation as a technical tool, so we shall not describe it in much detail. The necessary references can be found in Ref. [75].

At this point we remark that even for $N=1$ the instantons are present in the D-brane picture. They become visible in the gauge theory when noncommutativity is turned on. Remarkably, the actual value of the noncommutativity parameter $\Theta$ does not affect the expectation values of the 
chiral observables, thus simplifying our life enormously.

So far we presented the $\mathrm{D}$-brane realization of $\mathcal{N}=2$ theory. There exists another useful realization, via local Calabi-Yau manifolds, introduced in Ref. [70], which we already alluded to in the section 1.4. This realization, as we already explained in the introduction is useful in relating the prepotential to the topological string amplitudes. If the theory is embedded in the IIA string on local Calabi-Yau, then the interesting physics comes from the worldsheet instantons, wrapping some 2-cycles in the Calabi-Yau. In the mirror IIB description one gets a string without worldsheet instantons contributing to the prepotential, and effectively reducing to some field theory. This field theory is known in the case of global Calabi-Yau. But it is not known explicitly in the case of local Calabi-Yau. As we shall show, it can be sometimes identified with the free fermion theory on auxiliary Riemann surface (cf. Ref. [18]).

Relation to the geometrical engineering of Ref. [70] is also useful in making contact between our $\Omega$-deformation and the sugra backgrounds with graviphoton field strength. Indeed, our construction involved a lift to five or six dimensions. The first case embeds easily to IIA string theory where this corresponds to the lift to M-theory. To see the whole six dimensional picture (10) one should use IIB language and the lift to F-theory (one has to set $\bar{\Omega}=0$, though).

Let us consider the five dimensional lift. We have M-theory on the 11-fold with the metric:

$$
d s^{2}=\left(d x^{\mu}+\Omega_{\nu}^{\mu} x^{\nu} d \varphi\right)^{2}+r^{2} d \varphi^{2}+d s_{C Y}^{2}
$$

Here we assume, for simplicity, that $\epsilon_{1}=-\epsilon_{2}$, so that $\Omega=\Omega^{-}$generates an $S U(2)$ rotation, thus preserving half of susy. Now let us reduce on the circle $\mathbb{S}^{1}$ and interpret the background (19) in the type IIA string. Using Ref. [80] we arrive at the following IIA background:

$$
\begin{gathered}
g_{s}=\left(r^{2}+\|\Omega \cdot x\|^{2}\right)^{\frac{3}{4}} \\
A^{g r a v}=\frac{1}{r^{2}+\|\Omega \cdot x\|^{2}} \Omega_{\mu \nu} x^{\mu} d x^{\nu} \\
d s_{10}^{2}=\frac{1}{\sqrt{r^{2}+\|\Omega \cdot x\|^{2}}}\left(r^{2} d x^{2}+\Omega_{\nu}^{\mu} \Omega_{\kappa}^{\lambda}\left(x^{2} d x^{2} \delta^{\nu \kappa} \delta_{\mu \lambda}-x^{\nu} x^{\kappa} d x^{\mu} d x^{\lambda}\right)\right)+ \\
+\sqrt{r^{2}+\|\Omega \cdot x\|^{2}} d s_{C Y}^{2}
\end{gathered}
$$


where the graviphoton $U(1)$ field is turned on. The IIA string coupling becomes strong at $x \rightarrow \infty$. However, the effective coupling in the calculations of $\mathcal{F}_{g}$ is

$$
\hbar \sim g_{s} \sqrt{\left\|d A^{\text {grav }}\right\|^{2}} \sim\left(r^{2}+\|\Omega \cdot x\|^{2}\right)^{-\frac{1}{4}} \rightarrow 0, \quad x \rightarrow \infty
$$

\subsection{The partition function}

Our next goal is the calculation of the partition function

$$
Z\left(\tau_{\vec{n}} ; a, \Omega\right)=\int_{\phi(\infty)=a} D \Phi D A D \lambda \ldots e^{-S(\Omega)}
$$

of the $\mathcal{N}=2$ susy gauge theory with all the higher couplings (1) on the background (10) with the fixed asymptotics of the Higgs field at infinity. We use the fact that the chiral deformations are not sensitive to the anti-chiral parameters (up to holomorphic anomaly). We take the limit $\bar{\tau}_{0} \rightarrow \infty$, and the partition function becomes the sum over the instanton charges of the integrals over the moduli spaces $\mathfrak{M}$ of instantons of the measure, obtained by the developing the path integral perturbation expansion around instanton solutions.

On the other hand, if we take instead a low-energy limit, this calculation should reduce to that of low-energy effective theory. In the Seiberg-Witten story [65] the low-energy theory is characterized by the complexified energy scale $\Lambda \sim \mu_{U V} e^{2 \pi i \tau_{0}\left(\mu_{U V}\right)}$. We now recall Eq.( 14). In our setup the lowenergy scale is $(x, \theta)$-dependent:

$$
\Lambda(x, \theta)=\mu_{U V} e^{2 \pi i \tau_{0}\left(x, \theta ; \mu_{U V}\right)}=\Lambda e^{2 \pi i \bar{\Omega}^{-} \theta^{2}-\|\Omega \cdot x\|^{2}}
$$

Near $x=0$ it is finite, while at $x \rightarrow \infty$ the theory becomes infinitely weakly coupled. With Eq.( 14) in mind we can easily relate the partition function to the prepotential (5) (cf. Ref. [57]):

$$
\begin{gathered}
Z=Z^{p e r t} \exp \left[\int d^{4} x d^{4} \theta \mathcal{F}^{i n s t}\left(a ; \tau_{\vec{n}} ; \Lambda(x, \theta)\right)+\text { higher derivatives }\right]= \\
=\exp \frac{1}{\epsilon_{1} \epsilon_{2}}\left[\mathcal{F}\left(a, \tau_{\vec{n}} ; \Lambda\right)+O\left(\epsilon_{1}, \epsilon_{2}\right)\right]
\end{gathered}
$$

where $\mathcal{F}^{\text {inst }}$ is the sum of all instanton corrections to the prepotential, and $Z^{\text {pert }}$ is the result of the perturbative calculation on the $\Omega$-background. The corrections in $\epsilon_{1,2}$ come from the ignored higher derivative terms. 


\subsection{Perturbative part}

The perturbative part is given by the one-loop contribution from W-bosons, as well as non-zero angular momentum modes of the abelian photons (we shall comment on this below). Recall that in the $\Omega$-background one can integrate out all non-zero modes, as $\Omega$ lifts all massless fields. Because of the reduced supersymmetry the determinants do not quite cancel. The simplest way to calculate them is to go to the basis of normalizable spherical harmonics:

$$
\Phi=\sum_{l, m=1}^{N} T_{l m} \sum_{i, j, \bar{i}, \bar{j} \geq 1} \phi_{i j \bar{i} \bar{j}}^{l m} z_{1}^{i-1} z_{2}^{j-1} \bar{z}_{1}^{\bar{i}-1} \bar{z}_{2}^{\bar{j}-1} e^{-\left|z_{1}\right|^{2}-\left|z_{2}\right|^{2}}
$$

and similarly for the components of the gauge fields and so on. Here the terms with $l \neq m$ correspond to the $\mathrm{W}$-bosons, massive components of the Higgs field, and the massive components of the gluinos, while $l=m$ represent the abelian part. We are doing the WKB calculation around the trivial gauge field $A=0$ : the unborken susy guarantees there are no further corrections. The integral over the bosonic and fermionic fluctuations becomes a ratio of the determinants, formally:

$$
\prod_{l, m=1}^{N} \prod_{i, j=1}^{\infty} \prod_{i}^{\infty} \frac{\left(a_{l m}+\epsilon_{1}(i-\bar{i})+\epsilon_{2}(j-\bar{j})\right)\left(a_{l m}+\epsilon_{1}(i-\bar{i}-1)+\epsilon_{2}(j-\bar{j}-1)\right)}{\left(a_{l m}+\epsilon_{1}(i-\bar{i}-1)+\epsilon_{2}(j-\bar{j})\right)\left(a_{l m}+\epsilon_{1}(i-\bar{i})+\epsilon_{2}(j-\bar{j}-1)\right)}
$$

(recall that the "weight" of $A_{\mu}(z, \bar{z})$ has in addition to its "orbital" weight, which comes from the $(z, \bar{z})$-dependence, a spin $\left(-\epsilon_{1}\right)$ weight, similarly for $A_{\overline{2}}$ we have an extra $\left(-\epsilon_{2}\right)$, for $F^{0,2}$ extra $\left.\left(-\epsilon_{1}-\epsilon_{2}\right)\right)$. In the product over $\bar{i}, \bar{j}$ only the term with $\bar{i}=\bar{j}=1$ is not cancelled, giving rise to:

$$
Z^{p e r t}=\prod_{l, m ; i, j \geq 1}^{\prime}\left(a_{l}-a_{m}+\epsilon_{1}(i-1)+\epsilon_{2}(j-1)\right)
$$

times the conjugate term, which depends on $\bar{a}$. We shall ultimately take $\bar{a} \rightarrow \infty$, so we ignore this term - at any rate, it cancels out in the correlation functions of the chiral observables. The symbol $\Pi^{\prime}$ in Eq.( 27) means that the contribution of the abelian zero angular momentum modes $l=m, i=j=1$ to the product is omitted (this has to do with our boundary conditions). We shall always understand Eq.( 27) in the sense of $\zeta$-regularization. After regularization one can analytically continue to $\epsilon_{1}+\epsilon_{2}=0$. 
In fact, for $\epsilon_{1}=-\epsilon_{2}=\hbar$ one can expand:

$$
Z\left(\tau_{\vec{n}} ; a, \Omega\right)=\exp \left(-\sum_{g=0}^{\infty} \hbar^{2 g-2} \mathcal{F}_{g}\left(a ; \tau_{\vec{n}} ; \Lambda\right)\right)
$$

The higher "prepotentials" $\mathcal{F}_{g}$ turn out to be related to the higher genus string amplitudes, see Refs. [30,39,57].

\subsection{Mathematical realization of $\mathcal{N}=2$ theory}

The mathematical realization of the gauge theory we are studying is the following. Consider the space $\mathcal{Y}$ of all gauge fields on $\mathbb{R}^{4}$ with finite YangMills action. There are three groups of symmetries acting on this space which we shall study. The first group, $\mathcal{G}_{\infty}$, is the group of gauge transformations, trivial at infinity: $g(x) \rightarrow 1, x \rightarrow \infty$. The second, $G$ is the group of constant, global, gauge transformations. The group of all gauge transformations $\mathcal{G}$ is the extension of $\mathcal{G}_{\infty}$ by $G$, s.t. $G=\mathcal{G} / \mathcal{G}_{\infty}$. The third group $K=\operatorname{Spin}(4)$, is the covering group of the group of Euclidean rotations about some fixed point $x=0$. Over the space $\mathcal{Y}$ we consider the $\mathcal{G} \ltimes K$-equivariant vector bundle $\mathcal{V}$ of the self-dual two-forms on $\mathbb{R}^{4}$ with the values in the adjoint representation of the gauge group $\mathcal{G}$. For a gauge field $A \in \mathcal{Y}$ the self-dual projection of its curvature $F_{A}^{+}$defines a section of $\mathcal{V}$.

The path integral measure of the supersymmetric gauge theory with the extra $\Omega$-couplings is nothing but the Mathai-Quillen representative of the Euler class of $\mathcal{V}$, written using the section $F^{+}$, and working with $\mathcal{G} \ltimes K$ equivariantly. Calculating the path integral corresponds to the pushforward onto the quotient by the group $\mathcal{G}_{\infty}$ and its further localization w.r.t the remaining groups $G \times K$. The result is given by the sum over the fixed points of the $G \times K$ action on the moduli space of instantons $\mathfrak{M}$, i.e. solutions to $F^{+}=0$.

The chiral observables translate to the equivariant Chern classes of some natural bundles (sheaves) over the moduli space $\mathfrak{M}$. Their calculation is more or less standard and is presented in the next section.

\subsection{Nonperturbative part}

We now proceed with the calculation of the nonperturbative contribution to the partition function (22). There are two ways of determining it. One way is the direct analysis of the saddle points of the path integral measure. This is a nice excersize, but it relies on very explicit knowledge of the deformed instanton solutions $[16,54,59]$, invariant under the action of the group $K$ 
of rotations [10]. Instead, we shall choose slightly less explicit, but more general route.

The general property of the chiral observables in $\mathcal{N}=2$ theories, which is a direct consequence of the analysis in Ref. [78], is the cohomological nature of their correlation functions. Namely, in the limit $\bar{\tau}_{0} \rightarrow 0$ these become the integrals over the instanton moduli space $\mathfrak{M}$. The chiral observables, evaluated on the instanton collective coordinates, become closed differential forms. Thus, if the moduli space $\mathfrak{M}$ was compact and smooth, one could choose some convenient representatives of their cohomology classes to evaluate their integrals. Moreover, a generalization of the arguments in Ref. [78] allows to consider the $\mathcal{N}=2$ theory in the $\Omega$-background. In this case the differential forms on $\mathfrak{M}$ become $K$-equivariantly closed. Even though the space $\mathfrak{M}$ is not compact, the space of $K$-fixed points is, and this is good enough for the evaluation of the integrals of the $K$-equivariant integrals.

The final bit of information which makes the calculation of the chiral observables constructed out of the higher Casimirs possible, is the identification of the $K$-equivariantly closed differential forms on $\mathfrak{M}$ they represent with the densities of the equivariant Chern classes of some natural bundles over $\mathfrak{M}$. We now proceed with the explicit description of $\mathfrak{M}$, these natural bundles, and finally the chiral observables.

\section{ADHM construction}

To get a handle on these fixed point sets and to calculate the characteristic numbers of the various bundles we have defined above, we need to remind a few facts about the actual construction of $\mathfrak{M}$, the so-called ADHM construction, see Refs. [5,52]. In this construction one starts with two Hermitian vector spaces $W$ and $V$. One then looks for four Hermitian operators $\mathbb{X}^{\mu}: V \rightarrow V, \mu=1,2,3,4$ and two complex operators $\lambda_{\alpha}: W \rightarrow V, \alpha=1,2$ (and $\bar{\lambda}_{\dot{\alpha}}=\lambda_{\alpha}^{\dagger}: V \rightarrow W$ ), which can be combined into a sequence:

$$
0 \rightarrow W \otimes S_{-} \rightarrow V \oplus W \otimes S_{+} \rightarrow 0
$$

where the non-trivial map is given by:

$$
\mathcal{D}^{+}=\lambda \oplus \mathbb{X}^{\mu} \sigma_{\mu}
$$

The ADHM equation requires that $\mathcal{D D}^{+}$commutes with the Pauli matrices $\sigma_{\mu}$ acting in $S_{-}$. In addition, one requires that $\mathcal{D} \mathcal{D}^{+}$has a maximal rank. The moduli space $\mathfrak{M}$ is then identified with the space of such $\mathbb{X}, \lambda$ up to the action of the group $U(k)$ of unitary transformations in $V$. The group $G=U(N)$ acts on $\mathfrak{M}$ by the natural action, descending from that on $\lambda$ ( $\mathbb{X}$ 
are neutral). The group $K \approx \operatorname{Spin}(4)$ acts on $\mathfrak{M}$ by rotating $\mathbb{X}$ in the vector representation and $\lambda$ in the appropriate chiral spinor representation.

$D$-brane picture, again

The ADHM construction becomes very natural when the gauge theory is realized with the help of D-branes. The space $V$ is the Chan-Paton space for the $\mathrm{D}(-1)$ branes, while $W$ is the Chan-Paton space for the $\mathrm{D} 3$ branes. The matrices $\mathbb{X}$ are the ground states of the $(-1,-1)$ strings, while $\lambda_{\alpha}, \bar{\lambda}_{\dot{a}}$ are those of $(-1,3),(3,-1)$. The ADHM equations are the conditions for unbroken susy. Their solutions describe the Higgs branch of the $\mathrm{D}(-1)$ instanton theory ${ }^{\mathrm{b}}$. The $\mathrm{D}(-1)$ instantons also carry a multiplet responsible for the $U(V)$ "gauge" group. In particular, quantization of $(-1,-1)$ strings in addition to $\mathbb{X}$ gives rise to a matrix $\phi$ (not to be confused with $\Phi$ in the adjoint of $U(N)$ !) in the adjoint of $U(k)$, which represents the motion of $\mathrm{D}(-1)$ instantons in the directions, transverse to D3 branes.

Tangent and universal bundles.

The problem considered here is typical in the soliton physics. One finds some moduli space of solutions (collective coordinates) which should be quantized. The supersymmetric theories lead to supersymmetric quantum mechanics on the moduli spaces. If the gauge symmetry is present the collective coordinates are defined with the help of some gauge fixing procedure, which leads to the complications described below.

The tangent space to the instanton moduli space $\mathfrak{M}$ at the point $m$ can be described as follows. Pick a gauge field $A$ which corresponds to $m \in \mathfrak{M}$, $F^{+}(A)=0$. Any two such choices differ by a gauge transformation. Now consider deforming $A$ :

$$
A \rightarrow A+\delta A
$$

so that the new gauge field also obeys the instanton equation $F^{+}(A+\delta A)=$ 0 . In other words, $\delta A$ obeys the linear equations:

$$
\begin{aligned}
& D_{A}^{+} \delta A=0 \\
& D_{A}^{*} \delta A=0
\end{aligned}
$$

\footnotetext{
bo make this statements literally true one should consider D2-D6 system instead of D(-1)-D3 (to avoid off-shell string amplitudes, and the non-existence of moduli spaces of vacua in the field theories less then in three dimensions).
} 
where the first equation is the linearized anti-self-duality equation, while the second is the gauge choice, to project out the trivial deformations $\delta A \sim D_{A} \varepsilon$. Let us choose some basis in the (finite-dimensional) vector space of solutions to Eq. (30): $\delta A=a_{\mu}^{K} d x^{\mu} \zeta_{K}$, where $a^{K}$ obey Eq. (30), and, say, are orthonormal with respect to the natural metric $\left\langle a^{L} \mid a^{K}\right\rangle \equiv \int_{\mathbf{R}^{4}} a^{L} \wedge \star a^{K}=\delta_{L K}$, $L, K=1, \ldots, \operatorname{dim} \mathfrak{M}$. Now suppose we have a family of instanton gauge fields, parameterized by the points of $\mathfrak{M}: A_{\mu}(x ; m)$, where $x \in \mathbf{R}^{4}, m \in \mathfrak{M}$. Let us differentiate $A_{\mu}$ w.r.t the moduli $m$. Clearly, one can expand:

$$
\frac{\partial A}{\partial m^{L}}=a^{K} \zeta_{L K}+D_{A} \varepsilon_{L}
$$

The compensating gauge transformations $\varepsilon_{L}$ together with $A_{\mu}(m)$ form a connection $\mathcal{A}=A_{\mu}(x ; m) d x^{\mu}+\varepsilon_{L} d m^{L}$ in the rank $N$ vector bundle $\mathcal{E}$ over $\mathfrak{M} \times \mathbb{R}^{4}$. Now let us calculate its full curvature:

$$
\begin{gathered}
\mathcal{F}=\mathbf{d} \mathcal{A}+[\mathcal{A}, \mathcal{A}], \quad \mathbf{d}=d_{\mathfrak{M}}+d_{\mathbb{R}^{4}} \\
\mathcal{F}=\Phi+\Psi+F
\end{gathered}
$$

where $\Phi$ is a two-form on $\mathfrak{M}, \Psi$ is a one-form on $\mathfrak{M}$ and one-form on $\mathbb{R}^{4}$, and $F$ is a two-form on $\mathbb{R}^{4}$. The straightforward calculation shows that $\Phi, \Psi, F$ solve the equation:

$$
\Delta_{A} \Phi=[\Psi, \star \Psi], \quad D_{A}^{+} \Psi=0, \quad D_{A}^{*} \Psi=0, \quad F^{+}=0
$$

The equation on $\Phi$ is (up to $Q$-exact terms) identical to the equation on the adjoint Higgs field in the instanton background, while the equation on $\Psi$ is (again, up to $Q$-exact terms) identical to that on gluion zero modes. This relation between $\mathcal{F}$ and the chiral observables (which are, after all, the polynomials in $\Phi, \Psi, F$, up to $Q$-exact terms) will prove extremely useful in what follows. In particular, we can write:

$$
\begin{gathered}
\mathcal{O}_{J}^{(0)}=\frac{1}{J} \operatorname{Tr} \Phi^{J}, \quad \ldots \\
\mathcal{O}_{J}^{(4)}=\sum_{l=0}^{J-2} \operatorname{Tr}\left(\Phi^{l} F \Phi^{J-2-l} F\right)+ \\
+\sum_{l, n \geq 0, l+n \leq J-3} \operatorname{Tr}\left(\Phi^{l} F \Phi^{n} \Psi \Phi^{J-3-l-n} \Psi\right)+
\end{gathered}
$$




$$
+\sum_{l, k, n \geq 0, l+k+n \leq J-4} \operatorname{Tr}\left(\Phi^{l} \Psi \Phi^{k} \Psi \Phi^{n} \Psi \Phi^{J-4-k-l-n} \Psi\right)
$$

where we substitute the expressions for $\Phi, \Psi, F$ from Eq. 33 .

A mathematically oriented reader would object at this point, as it is well-known that universal bundles together with a nice connections do not exist over the compactified moduli spaces. We shall not pay attention to these (fully just) remarks, as eventually there is a way around. We find it more straightforward to explain things as if such objects existed over the compactified moduli space of instantons. Let $p$ denote the projection $\mathfrak{M} \times \mathbf{R}^{4} \rightarrow \mathfrak{M}$. Suppose we know everything about $\mathcal{E}$. How would we reconstruct $T \mathfrak{M}$ from there? We know already that the tangent space to $\mathfrak{M}$ at a point $m$ is spanned by the solutions to Eq. (30). It is plain to identify these solutions with the cohomology of the Atiyah-Singer complex:

$$
0 \longrightarrow \Omega^{0}\left(\mathbf{R}^{4}\right) \otimes \mathfrak{g} \longrightarrow \Omega^{1}\left(\mathbf{R}^{4}\right) \otimes \mathfrak{g} \longrightarrow \Omega^{2,+}\left(\mathbf{R}^{4}\right) \otimes \mathfrak{g} \longrightarrow 0
$$

where the first non-trivial arrow is the infinitesimal gauge transformation: $\varepsilon \mapsto D_{A} \varepsilon$ and the second it $\delta A \mapsto D_{A}^{+} \delta A$. Thanks to $F_{A}^{+}=0$ this is indeed a complex, i.e. $D_{A}^{+} D_{A}=0$. The spaces $\Omega^{k} \otimes \mathfrak{g}$ can be viewed as the bundles over $\mathfrak{M} \times \mathbf{R}^{4}$, e.g. for $G=U(N)$

$$
\Omega^{k}\left(\mathbf{R}^{4}\right) \otimes \mathfrak{g}=\mathcal{E} \otimes \mathcal{E}^{*} \otimes \Lambda^{k} T^{*} \mathbf{R}^{4}
$$

Generically the complex(37) has only $H^{1}$ cohomology. We are thus led to identify K-classes: $T \mathfrak{M}=H^{1}-H^{0}-H^{2}$.

Framing and Dirac bundles.

We shall need two more natural bundles over $\mathfrak{M}$. As $\mathfrak{M}$ is defined by the quotient w.r.t. the group of gauge transformations, trivial at infinity, we have a bundle $W$ over $\mathfrak{M}$ whose fiber is the fiber of the original $U(N)$ bundle over $\mathbf{R}^{4}$ at infinity. Another important bundle is the bundle $V$ of Dirac zero modes. Its fiber over the point $m \in \mathfrak{M}$ is the space of normalizable solutions to the Dirac equation in fundamental representation in the background of the instanton gauge field, corresponding to $m$. In $K(\mathfrak{M})$,

$$
W=\left.\lim _{x \rightarrow \infty} \mathcal{E}\right|_{x}, \quad V=p_{*} \mathcal{E}
$$

The pushforward $p_{*}$ is defined here in $L^{2}$ sense. In what follows we shall need its equivariant analogue. Finally, let $S_{ \pm}$denote the bundles of positive and negative chirality spinors over $\mathbb{R}^{4}$. These bundles are trivial topologically. However they are nontrivial as $K$-equivariant bundles. 
Relations among bundles.

We arrive at the following relation among the virtual bundles:

$$
\begin{gathered}
\mathcal{E}=W \oplus V \otimes\left(S_{+}-S_{-}\right) \\
T \mathfrak{M}=-p_{*}\left(\mathcal{E} \otimes \mathcal{E}^{*}\right)
\end{gathered}
$$

The chiral operators $\mathcal{O}_{\vec{n}}$ we discussed in the introduction now are in oneto-one correspondence with the characteristic classes of the $U(N)$ bundles. A convenient basis in the space of such classes is given by the skew Schur functions, labelled by the partitions $\lambda=\left(\lambda_{1} \geq \lambda_{2} \geq \ldots \lambda_{N} \geq 0\right)$ :

$$
\mathbf{c h}_{\lambda}=\operatorname{Det}\left\|c h_{\lambda_{i}-i+j}\right\|
$$

Another basis is labelled by finite sequences $n_{1}, n_{2}, \ldots, n_{k}$ of non-negative integers:

$$
\mathcal{O}_{\vec{n}}=\prod_{J=1}^{\infty} \frac{1}{n_{J} !}\left(\frac{c h_{J}}{J}\right)^{n_{J}}
$$

It is this basis that we used in Eq. (1).

The Eqs. (40) imply some relations among the Chern classes, which we shall use. It is convenient to discuss the Chern characters first. Recall that we always work $G \times K$-equivariantly.

We get:

$$
\begin{gathered}
C h(\mathcal{E})=C h(W)+C h(V) \prod_{i=1}^{2}\left(e^{\frac{x_{i}}{2}}-e^{-\frac{x_{i}}{2}}\right) \\
C h(T \mathfrak{M})=-\int_{\mathbb{R}^{4}} C h(\mathcal{E}) C h\left(\mathcal{E}^{*}\right) \prod_{i=1}^{2}\left(\frac{x_{i}}{e^{\frac{x_{i}}{2}}-e^{-\frac{x_{i}}{2}}}\right)
\end{gathered}
$$

where $x_{1}, x_{2}$ are the equivariant Chern roots of the tangent bundle to $\mathbb{R}^{4}$ :

$$
x_{i}=\epsilon_{i}+\mathcal{R}_{i}
$$

where $\mathcal{R}_{i}=\frac{1}{2 \pi i} \delta^{2}\left(z_{i}\right) d z_{i} \wedge d \bar{z}_{i}$ is a curvature two-form ${ }^{\mathrm{c}}$ on $\mathbb{R}^{4}$. As everything is $K$-equivariant, the integral over $\mathbb{R}^{4}$ localizes onto the $K$-fixed point, the

\footnotetext{
${ }^{\mathrm{c}}$ For those worried by the singular form of Eq. (44), here is a nonsingular representative. Choose a smooth function $f(r)$ which is equal to 1 for sufficiently large $r$, and vanishes at $r=0$. Then $x_{i}$ is $K$-equivariantly cohomologous to $\epsilon_{i} f\left(\left|z_{i}\right|^{2}\right)+\frac{1}{2 \pi} f^{\prime}\left(\left|z_{i}\right|^{2}\right) d z_{i} \wedge d \bar{z}_{i}$.
} 
origin, as is also clear from the explicit formula Eq. (44):

$$
C h(T \mathfrak{M})=-\left[C h(\mathcal{E}) C h\left(\mathcal{E}^{*}\right)\right]_{0} \prod_{i=1}^{2}\left(\frac{1}{e^{\frac{\epsilon_{i}}{2}}-e^{-\frac{\epsilon_{i}}{2}}}\right)
$$

where $\left[C h(\mathcal{E}) C h\left(\mathcal{E}^{*}\right)\right]_{0}$ is the evaluation of the product of the Chern characters at the origin of $\mathbb{R}^{4}$.

\section{Integration over $\mathfrak{M}$}

Now we want to integrate over $\mathfrak{M}$. Suppose the integrand is the $\mathbb{G}=$ $G \times K$-equivariant differential form (see appendix A for definitions) $\Omega_{\mathcal{O}}[\varphi]$, $\varphi \in \operatorname{Lie}(\mathbb{G})$. Such integrals can be computed using localization. In plain words it means that there are given by the sums over the fixed points of the action of the one-parametric subgroup $\exp (t \mathfrak{a}), t \in \mathbb{R}$, of $\mathbb{G}, \mathfrak{a} \in \operatorname{Lie}(\mathbb{G})$. The contribution of each fixed point $P \in \mathfrak{M}$ (assuming it is isolated and $\mathfrak{M}$ is smooth at this point) is given by the ratio:

$$
Z_{P}=\frac{\left.\Omega_{\mathcal{O}}[\mathfrak{a}]^{(0)}\right|_{P}}{\left.c(T \mathfrak{M})[\mathfrak{a}]^{(0)}\right|_{P}}
$$

where $\omega^{(0)}$ denotes the scalar component of the inhomogeneous differential form corresponding to the equivariant differential form $\omega$, and $c(T \mathfrak{M})$ is the equivariant Chern polynomial of $T \mathfrak{M}$. It is defined as follows. As $T \mathfrak{M}$ is $\mathbb{G}$-equivariant, with respect to the maximal torus $\mathbb{T}$ it splits as a direct sum of the line bundles, $T \mathfrak{M}=\bigoplus_{i} L_{i}$, on which $\mathfrak{t}$ acts with some weight $w_{i}$ (a linear function on $\mathfrak{t}$ ). The equivariant Chern polynomial is defined simply by:

$$
c(T \mathfrak{M})[\mathfrak{a}]=\prod_{i}\left(c_{1}\left(L_{i}\right)+w_{i}(\mathfrak{a})\right)
$$

In order to proceed we need to calculate the numerator and the denominator of Eq. (46) and to sum over the points $P$. We need first the equivariant Chern polynomial $c(T \mathfrak{M})$. We already have an expression Eq. (45) for the equivariant Chern character of $T \mathfrak{M}$. To use it we recall that in terms of $L_{i}$ 's:

$$
C h(T \mathfrak{M})=\sum_{i} e^{c_{1}\left(L_{i}\right)+w_{i}(\mathfrak{a})}
$$

so that if (48) is known then so is (47). Moreover, if the fixed points $P$ are isolated (and they will be), the actual first Chern classes of $L_{i}$ will never contribute (they are two-forms and we simply want to evaluate Eqs. $(48,47)$ at a point $P$ ), so we only need to find $w_{i}$ 's - the weights. 
Now, what about $\Omega_{\mathcal{O}}$ ? Well, we construct it using the descendents of the Casimirs $\operatorname{Tr} \Phi^{J}$ and their multi-trace products. As we explained above, these become the polynomials in the traces of the powers of the universal curvature $\mathcal{F}$ as in Eq. (35). That is to say, they are cohomologous to the Chern classes of the universal bundle $\mathcal{E}$.

We are mostly interested in the correlators of the 4-descendents $\mathcal{O}^{(4)}$ of the invariant polynomials $\mathcal{P}(\Phi)$ on $\operatorname{Lie}(G)$. On the moduli space $\mathfrak{M}$ these are cohomologous to the integrals over $\mathbb{R}^{4}$ of the polynomials in the Chern classes $\operatorname{ch}_{k}(\mathcal{E})$ of the universal bundle. Again, thanks to $\mathbb{G}$-equivariance, these integrals are simply given by the localization at the origin in $\mathbb{R}^{4}$ :

$$
\mathcal{O}_{\mathcal{P}}^{(4)}=\frac{[\mathcal{P}(\mathcal{F})]_{0}}{\epsilon_{1} \epsilon_{2}}
$$

For $\mathcal{P}_{k}(\Phi)=\frac{1}{(2 \pi i)^{k} k !} \operatorname{Tr} \Phi^{k}, \mathcal{P}_{k}(\mathcal{F})=c h_{k}(\mathcal{E})$. Any other invariant polynomial is a polynomial in these $\mathcal{P}_{k}$.

\section{Evaluation of Chern classes at fixed points}

So, we see that everything reduces to the enumeration of the fixed points $P$, and the evaluation of the Chern classes of $\mathcal{E}$ at these points. Moreover, thanks to Eq. (48) it is sufficient to evaluate the restriction of $C h(W)$ and $C h(V)$.

These problems were solved in Ref. [57] for any $N$ using the results of Ref. [52] for $N=1$. The result is the following. The fixed points are in one-to-one correspondence with the $N$-tuples of partitions: $\overrightarrow{\mathfrak{k}}=\left(\mathfrak{k}_{1}, \ldots, \mathfrak{k}_{N}\right)$, where

$$
\mathfrak{k}_{l}=\left(k_{l 1} \geq k_{l 2} \geq k_{l 3} \geq \ldots k_{l n_{l}}>k_{l n_{l}+1}=0 \ldots\right)
$$

At the fixed point $P_{\overrightarrow{\mathfrak{k}}}$ corresponding to such an $N$-tuple, the Chern characters of the bundles $W$ and $V$ evaluate to:

$$
\begin{gathered}
{[C h(W)]_{P_{\overrightarrow{\mathfrak{k}}}}=\sum_{l=1}^{N} e^{a_{l}}} \\
{[C h(V)]_{P_{\overrightarrow{\mathfrak{k}}}}=\sum_{l=1}^{N} \sum_{i=1}^{n_{l}} \sum_{j=1}^{k_{l i}} e^{a_{l}+\epsilon_{1}(i-1)+\epsilon_{2}(j-1)}}
\end{gathered}
$$

From this we derive an expression for $C h(\mathcal{E})$, and for $c(T \mathfrak{M})$.

D-brane picture of partitions 
It is useful to recall here the D-brane interpretation of the partitions $\mathfrak{k}$. In this picture, the fractional D3-branes are separated in the $w$ direction, and are located at $w=a_{l}, l=1, \ldots, N$. To the l'th D3 brane $k_{l} \mathrm{D}(-1)$ instantons $\left(k_{l}=\sum_{i} k_{l i}\right)$ are attached. In the noncommutative theory with the noncommutativity parameter $\Theta$,

$$
\left[x^{1}, x^{2}\right]=\left[x^{3}, x^{4}\right]=i \Theta
$$

these $\mathrm{D}(-1)$ instantons are located near the origin $\left(z_{1}, z_{2}\right) \sim 0$, where $z_{1}=$ $x^{1}+i x^{2}, z_{2}=x^{3}+i x^{4}$. Different partitions correspond to the different 0 dimensional "submanifolds" (in the algebraic geometry sense) of $\mathbb{C}^{2}$. If we denote by $\mathcal{I}_{l}$ the algebra of holomorphic functions (polynomials) on $\mathbb{C}^{2}$ which vanish on the $\mathrm{D}(-1)$ instantons, stuck to the l'th $\mathrm{D} 3$-brane, then it can be identified with the ideal in the ring of polynomials $\mathbb{C}\left[z_{1}, z_{2}\right]$ such that the quotient $\mathbb{C}\left[z_{1}, z_{2}\right] / \mathcal{I}_{l}$ is spanned by the monomials

$$
z_{1}^{i-1} z_{2}^{j-1}, \quad 1 \leq j \leq k_{l i}
$$

Remark on Planck constant

In what follows we set $\epsilon_{1}=-\epsilon_{2}=\hbar$. Note, that this Planck constant has nothing to do with the coupling constant of the gauge theory, where it appears as the parameter of the geometric background (10). It corresponds however exactly to the loop counting in the dual string theory, while the gauge theory Planck constant in string theory picture arises as a worldsheet parameter, according to the relation between the world-sheet and gauge theory instantons, described in Ref. [39].

\subsection{Correlation functions of the chiral operators}

Now we are ready to attack the correlation function in Eq. (5). First of all, using the unbroken supercharges one argues that this correlation function is independent of the coefficient in front of the term $\left|F^{+}\right|^{2}+\ldots$ which is $\{Q, \ldots\}$. Therefore, one can go to the weak coupling regime (with the theta angle appropriately adjusted, so that $\tau_{0}$ is finite, while $\left.\bar{\tau}_{0} \rightarrow \infty\right)$ in which the expression in Eq. (5) is saturated by instantons (cf. Ref. [60]).

In this limit the descendants of the chiral operators become the Chern classes of the universal bundle, "integrated" (in the equivariant sense), over $\mathbf{R}^{4}$. Here is the table of equivariant integrals Ref. 22, cf. Eq. (24):

$$
\int_{\mathbf{R}^{4}} \Omega^{(4)}=\frac{\Omega^{(0)}(0)}{\epsilon_{1} \epsilon_{2}}
$$


We should then integrate these classes over $\mathfrak{M}$. But then again, we use equivariant localization, this time on the fixed points in $\mathfrak{M}$. These fixed points are labelled by partitions $\mathfrak{k}$. The calculation of the expectation values of the chiral operators becomes equivalent to the calculation of the expectation values of some operators in the statistical mechanical model, where the basic variables are the $N$-tuples of partitions (50). In this statistical model, the operator $\mathcal{O}_{J}^{(0)}=\frac{1}{J} \operatorname{Tr} \Phi^{J}$ in the gauge theory translates to the operator $\left(a_{l}=\hbar M_{l}\right)$ :

$$
\begin{gathered}
\mathcal{O}_{J}[\overrightarrow{\mathfrak{k}}] \equiv\left[\int_{\mathbb{R}^{4}} \mathcal{O}_{J}^{(4)}\right]_{P_{\overrightarrow{\mathfrak{k}}}}=\frac{\hbar^{J}}{J} \times \\
\sum_{l=1}^{N}\left[M_{l}^{J}+\left(\sum_{i=1}^{\infty}\left(M_{l}+k_{l i}-i+1\right)^{J}-\left(M_{l}+k_{l i}-i\right)^{J}-\left(M_{l}+1-i\right)^{J}+\left(M_{l}-i\right)^{J}\right)\right] \\
\text { formally } \frac{1}{J} \sum_{l, i}\left[\left(\left(a_{l}+\hbar\left(k_{l i}+1-i\right)\right)^{J}-\left(a_{l}+\hbar\left(k_{l i}-i\right)\right)^{J}\right]\right.
\end{gathered}
$$

This is a straightforward consequence of Eq. (51) for $\epsilon_{1}=-\epsilon_{2}=\hbar$.

Given the single-trace operators $\mathcal{O}_{J}$ we build arbitrary gauge-invariant operators $\mathcal{O}_{\vec{n}}$ as in Eqs. $(1,6)$. After that one can integrate their $\mathcal{N}=2$ descendants $\mathcal{O}_{\vec{n}}^{(4)}$ using the table of equivariant integrals (52).

Gauge theory generating function of the correlators of the chiral operators becomes the statistical model partition function with all the integrated operators $\int_{\mathbb{R}^{4}} \mathcal{O}_{\vec{n}}^{(4)}$ added to the Hamiltonian. In other words, we sum over the partitions $\left\{\mathbf{k}_{l}\right\}=\left\{k_{l i}\right\}$ the Bolzmann weights $\exp \left(-\frac{1}{\hbar^{2}} \sum_{\vec{n}} t_{\vec{n}} \mathcal{O}_{\vec{n}}\right)$, and the measure on the partitions is given by the square of the regularized discretized Vandermonde determinant:

$$
\begin{gathered}
\mu_{\overrightarrow{\mathfrak{k}}}=\prod_{(l i) \neq(m j)}\left(\lambda_{l i}-\lambda_{m j}\right) \\
\lambda_{l i}=a_{l}+\hbar\left(k_{l i}-i\right),
\end{gathered}
$$

The product in Eq. (54) is taken over all pairs $(l i) \neq(m j)$ which is short for $\{(l \neq m)$; or $(l=m, i \neq j) ;\}$ and can be understood with the help of $\zeta$-regularization:

$$
\mu_{\overrightarrow{\mathfrak{k}}}=\exp \left(-\left.\frac{d}{d s} \frac{1}{\Gamma(s)} \int_{0}^{\infty} d t t^{s-1} \sum_{(l i) \neq(m j)} e^{-t\left(\lambda_{l i}-\lambda_{m j}\right)}\right|_{s=0}\right)
$$


The sum in Eq. (55) is defined by analytic continuation, as the sum over $(l, i)$ converges for $\operatorname{Re}(\hbar t)<0$, while the sum over $(m, j)$ converges for $\operatorname{Re}(\hbar t)>0$.

\subsection{Full partition function on $\mathbb{R}^{4}$}

In order to proceed with more general four-manifolds we need the partition function on $\mathbb{R}^{4}$ to be evaluated for the most general parameters, both toric $\epsilon_{1}, \epsilon_{2}$ and the couplings $\tau_{\vec{n}}$. The discussion in the previous sections generalizes to:

$$
Z\left(\vec{a}, \epsilon_{1}, \epsilon_{2} ; \tau_{\vec{n}}\right)=\exp \frac{\mathcal{F}_{0}}{\epsilon_{1} \epsilon_{2}}+\frac{\epsilon_{1}+\epsilon_{2}}{\epsilon_{1} \epsilon_{2}} \mathcal{H}_{\frac{1}{2}}+\mathcal{F}_{1}+\frac{\left(\epsilon_{1}+\epsilon_{2}\right)^{2}}{\epsilon_{1} \epsilon_{2}} \mathcal{G}_{1}+\mathcal{O}\left(\epsilon_{1}, \epsilon_{2}\right)
$$

where $\mathcal{F}_{0}, \mathcal{F}_{1}, \mathcal{H}_{\frac{1}{2}}, \mathcal{G}_{1}$ are functions of $\vec{a}, \tau_{\vec{n}}$ (see Ref. [53] for a thorough discussion of these terms)

\section{GAUGE THEORY ON FOUR-MANIFOLDS}

Once we have learned how to deal with instantons on Euclidean space, it makes sense to generalize to more general four dimensional manifolds. One immediate obstacle to get a soluble theory is the supersymmetry breaking. Indeed, the supersymmetry of the $\mathcal{N}=2$ gauge theory is generated by the spinorial supercharges. On the curved manifold the supersymmetry transformation would lead to the variation of gravitino, unless the spinor $\varepsilon_{\alpha}$, which generates it, is covariantly constant, $\nabla_{\mu} \varepsilon_{\alpha}$. This is not possible for generic metric. However, one can make use of the extended nature of the supersymmetry, more precisely of the presence of the global symmetry group $S U(2)_{I}$, which allows to compensate for the non-covariance of the spinor, by its simultaneous $S U(2)_{I}$ transformation:

$$
\nabla_{\mu} \varepsilon_{\alpha}^{i}+A_{\mu, j}^{i} \varepsilon_{\alpha}^{j}=0, \quad \nabla_{\mu} \bar{\varepsilon}_{\dot{\alpha}}^{i}+A_{\mu, j}^{i} \bar{\varepsilon}_{\dot{\alpha}}^{j}=0
$$

where $A_{\mu, j}^{i}$ is the background $S U(2)_{I}$ gauge field. If it is chosen to be the opposite of the $S U(2)_{L}$ or $S U(2)_{R}$ component of the spin connection, then the Eq. (57) has solutions, for constant $\varepsilon_{\alpha}^{i} \sim \delta_{\alpha}^{i}$ or $\bar{\varepsilon}_{\dot{\alpha}}^{i} \sim \delta_{\dot{\alpha}}^{i}$ respectively. This procedure of coupling the R-symmetry to the background metric is called twisting, it was introduced by E. Witten in his field theoretic interpretation of Donaldson theory, Ref. [78], and was later generalized to sigma models.

The twisting effectively changes the Lorentz spins of the supercharges. Instead of the spinors, they become: a scalar $\mathcal{Q}$, a self-dual two-form $\mathcal{Q}_{\mu \nu}^{+}$ and a one-form $G_{\mu}$. We shall not write down the obvious anti-commutation relations, see Ref. $[39,56,57]$ for details. 
The generic metric on the curved space will only support the scalar $\mathcal{Q}$ as an unbroken supercharge. It squares to zero up to gauge transformations, and leads to the set of chiral operators which are identified in Ref. [78] with Donaldson $\mu$-classes (in the case of $S U(2)$ gauge group).

If the four-manifold is Kähler, then in addition to $\mathcal{Q}$, the charge $\mathcal{Q}_{\mu \nu}^{+} \omega^{\mu \nu}$ is conserved, where $\omega$ is the Kahler two-form. If it is hyperkähler, then one gets three additional supercharges, at the price of one: $\mathcal{Q}_{A}=\mathcal{Q}_{\mu \nu}^{+} \omega_{A}^{\mu \nu}, A=1,2,3$, because on hyperkähler space one has, by definition, a triplet of covariantly constant symplectic forms. These properties of twisted superalgebra were exploited in Ref. [79].

We shall exploit another possibility. Namely, if the manifold has isometry generated by the vector field $V^{\mu}, \nabla_{\nu} V_{\mu}+\nabla_{\mu} V_{\nu}=0$, then the twisted theory posesses another conserved supercharge, $V^{\mu} G_{\mu}$. By combining it with the standard one, $\mathcal{Q}$, one arrives at the multi-parametric family of supercharges:

$$
\tilde{\mathcal{Q}}(t)=\mathcal{Q}+\varepsilon^{\ell} V_{\ell}^{\mu} G_{\mu}
$$

where the index $\ell$ runs through the set of all isometries, and $\varepsilon^{\ell}$ are some parameters. Note that in Eq. (58) we take a linear combination of operators with position-dependent coefficients. In order for them to form a closed algebra the vector fields $V_{\ell}^{\mu}$ must form an algebra. Happily they do. In what follows we shall only talk about the maximally abelian subgroup of the group of isometries, for which $\left[V_{\ell}, V_{\ell^{\prime}}\right]=0$.

\section{1. $\Omega$-background: global case}

We are now in position to explain what should one do to the gauge theory in order to exhibit the symmetry with respect to the supercharges $\tilde{\mathcal{Q}}$. We should subject the theory to the $\Omega$-background.

The standard Donaldson theory in four dimensions can be viewed as the partially twisted six dimensional super-Yang-Mills theory, compacitifed on $\mathbb{T}^{2} \times X$, where $X$ is our four-fold, and the torus $\mathbb{T}^{2}$ has vanishing size.

Now, for $X$ which has isometries, we modify this construction. Namely, we consider the flat $X$-bundle over $\mathbb{T}^{2}$, which can be viewed as an orbifold of $\mathbb{R}^{2} \times X$ by the action of $\mathbb{Z}^{2}$, which acts by shifts in $\mathbb{R}^{2}$ direction, and by two commuting isometries $g_{1}, g_{2}$ in the $X$ direction:

$$
(z, \bar{z}, x) \mapsto\left(z+n+m \tau, \bar{z}+n+m \bar{\tau}, g_{1}^{n} g_{2}^{m} \cdot x\right)
$$

By compactifying the gauge theory on this background, with the partial twist along $X$, in the limit of vanishing volume of $\mathbb{T}^{2}$ we end up with the 
theory:

$$
\begin{gathered}
S^{b o s}=\int_{X} \operatorname{tr} F \wedge \star F+\operatorname{tr}\left(D_{A} \Phi-L_{V} F\right) \wedge \star\left(D_{A} \bar{\Phi}-L_{\bar{V}} F\right)+ \\
+\operatorname{tr}\left([\Phi, \bar{\Phi}]+\iota_{V} D_{A} \bar{\Phi}-\iota_{\bar{V}} D_{A} \Phi\right)^{2}+\ldots
\end{gathered}
$$

where ... stand for fermionic terms, and $V=\varepsilon^{\ell} V_{\ell}$.

We shall now study the theory ( 60 ) on toric varieties.

\subsection{Toric preliminaries}

In this section we collect all the necessary information about toric varieties. We use slightly unconventional language, which is more familiar to physicists.

Let $M, d$ be non-negative integers, and $\mathbb{T}^{M}, \mathbb{T}^{d}, \mathbb{T}^{M+d}$ the standard tori $U(1)^{M, d, M+d}$ of the corresponding dimensions. In what follows $i$ runs from 1 to $M+d, a$ runs from 1 to $M$ and $\mu$ runs from 1 to $d$. The torus $\mathbb{T}^{M+d}$ acts in the standard fashion on the space $\mathbb{C}^{M+d}$ :

$$
Z=\left(Z^{i}\right) \mapsto\left(Z^{i} \exp i \theta^{i}\right)
$$

Any integral matrix $Q: \mathbb{Z}^{M} \rightarrow \mathbb{Z}^{M+d}$ defines a homomorphism of $\mathbb{T}^{M}$ into $\mathbb{T}^{M+d}$, and the corresponding action of $\mathbb{T}^{M}$ on $\mathbb{C}^{M+d}$ :

$$
Z=\left(Z^{i}\right) \mapsto\left(Z^{i} \exp i \sum_{a} Q_{a}^{i} \varphi^{a}\right)
$$

This action preserves the norms: $p_{i}=\frac{1}{2}\left|Z^{i}\right|^{2}$ and the symplectic form

$$
\omega=\sum_{i} d p_{i} \wedge d \vartheta^{i} \quad Z^{i}=\sqrt{2 p_{i}} e^{i \vartheta^{i}}
$$

Now, fix a collection $\vec{r}$ of $M$ real numbers $r_{1}, \ldots, r_{M}$ - Fayet-Illiopoulos terms in the physical language - and consider the Hamiltonian reduction of $\mathbb{C}^{M+d}$ with respect to $\mathbb{T}^{M}$ at the level of the moment map

$$
\overrightarrow{\mathbf{m}}=\left(\mathbf{m}_{1}, \ldots, \mathbf{m}_{M}\right), \quad \mathbf{m}_{a}=\sum_{i} Q_{a}^{i} p_{i}
$$

given by $\vec{r}$. Explicitly, we consider the space

$$
X=\overrightarrow{\mathbf{m}}^{-1}(\vec{r}) / \mathbb{T}^{M}
$$

This is our toric variety.

By construction, it comes endowed with the map:

$$
\vec{p}: X \rightarrow \mathbb{R}_{+}^{M+d}
$$


which factors through the projection

$$
X \rightarrow X / \mathbb{T}^{d}=\Delta_{X}
$$

where $\mathbb{T}^{d}=\mathbb{T}^{M+d} / \mathbb{T}^{M}$. We neglect possible torsion part (it is absent for generic $Q$ ).

The image of $\Delta_{X}$ under $\vec{p}$ is the convex $d$-dimensional polyhedron (perhaps non-compact), which fits some $d$-dimensional affine subspace $b L^{d}$ in $\mathbb{R}_{+}^{M+d}$.

Another canonical structure on $X$ is a set of complex line bundles $L_{a}$ and $\mathcal{L}_{i}$. More precisely, any character $\chi$ of the torus $\mathbb{T}^{M}$ defines a line bundle, which is associated with the help of $\chi$ to the principal $\mathbb{T}^{M}$ bundle:

$$
\overrightarrow{\mathbf{m}}^{-1}(\vec{r}) \rightarrow X
$$

All of the above is said under the assumption of genericity of $\vec{r}$, so that the Eq. (61) indeed defines a principal bundle. The line bundles $\mathcal{L}_{i}$ are associated to the characters $\chi_{i}^{M}=\exp i Q_{a}^{i} \varphi^{a}$, while $L_{a}$ are associated to $\chi_{a}^{M}=\exp i \varphi^{a}$. Hence, topologically:

$$
\mathcal{L}_{i} \approx \otimes_{a} L_{a}^{\otimes Q_{a}^{i}}
$$

The torus $\mathbb{T}^{M+d}$ acts on $X$. Of course, only $\mathbb{T}^{d}$ acts faithfully. The full torus $\mathbb{T}^{M+d}$ becomes visible when we start looking at the line bundles over $X$. Then $\mathcal{L}_{i}$ become distinct line bundles as $\mathbb{T}^{M+d}$ equivariant bundles. They correspond to the characters $\chi_{i}^{M+d}=e^{i \theta^{i}}$. In what follows we shall denote $\chi^{M+d}$ simply by $\chi$.

The fixed points $v$ of $\mathbb{T}^{d}$ action on $X$ are the vertices of $\Delta_{X}$. Generically, these vertices have $d$ edges emanating from them. These edges correspond to fixed complex projective lines in $X$ (if $X$ is non-compact the edges may go all the way to infinity, in this case they correspond to the fixed complex lines in $X$ ).

The tangent space $T_{v} X$ to $X$ at any fixed point is a representation of $\mathbb{T}^{d}$, and, consequently, a representation of $\mathbb{T}^{M+d}$. The representation splits as a sum of $d$ one-dimensional irreducibles:

$$
T_{v} X=\bigoplus_{\mu=1}^{d} \chi_{v, \mu}
$$

where we took the liberty of identifying the characters with the one dimensional representations. The characters $\chi_{v, \mu}$ define the so-called weights (we have seen their analogues in the perturbative formula Eq. (26)) of the $\mathbb{T}^{M+d}$ 
action on $T_{v} X$ :

$$
\chi_{v, \mu}=\exp i \sum_{i} w_{v, \mu ; i} \theta^{i}
$$

which clearly obey:

$$
\sum_{i} w_{v, \mu ; i} Q_{a}^{i}=0, \forall a
$$

Each fixed point $v$ defines a subset $I_{v}$ of the set of indices $\{1, \ldots, M+d\}$ of cardinality $d$, such that for any

$$
\forall i \in I_{v} \quad Z^{i}(v)=0
$$

It is natural to label the elements of $I_{v}$ by the index $\mu=1, \ldots, d$. In other words, $I_{v}$ coincides with the set of weight subspaces of $T_{v} X$.

The remaining $M$ coordinates $Z^{i}$ are completely fixed by the moment map equations, up to the action of $\mathbb{T}^{M}$. Let $\mathbb{Z}_{v}^{M} \subset\left(\mathbb{Z}^{M+d}\right)^{t}$ denote the sub-lattice $\left\{\left(n_{1}, \ldots, n_{M+d}\right) \mid n_{i}=0, i \in I_{v}\right\}$, and $\mathbb{R}_{v}^{M}=\mathbb{Z}_{v}^{M} \otimes \mathbb{R}$. Then $\vec{\partial}(v) \in \mathbb{R}_{v}^{M}$. Let $Q_{v}$ denote the restriction of $Q^{t}$ onto $\mathbb{Z}_{v}^{M}$. It must be invertible over $\mathbb{Z}$ in order for $v$ to be isolated fixed point. Then:

$$
w_{v, \mu ; i}=\left(\delta_{\mu ; i}-Q_{a}^{\mu}\left(Q_{v}^{-1}\right)_{i}^{a}\right)
$$

Second cohomology The line bundles $L_{a}$ generate the $K$-group of $X$. Their

first Chern classes generate $H^{2}(X, \mathbb{Z})$. In the $\mathbb{T}^{M+d}$-equivariant cohomology they are represented by the equivariantly closed forms: $\phi_{a}+c_{1}\left(L_{a}\right)$. In the localized cohomology, which is spanned by the fixed points $v \in X^{\mathbb{T}}$, each point contributing a copy of $\mathbb{C}\left(\left(\varepsilon_{1}, \ldots, \varepsilon_{M+d}\right)\right)$, the Chern classes localized to $\phi_{a}^{(v)}$, which are linear functions of $\varepsilon_{i}$.

In the applications of gauge theory to four-manifold invariants one studies correlators of the 2-observables, say for single trace operators: $\mathcal{O}_{J}^{(2)}$, see Eqs. $(35,36)$. The integrated 2-observables are evaluated using localization:

$$
\int_{\Sigma} \mathcal{O}_{J}^{(2)}=\sum_{v} H_{\Sigma}^{(v)} C h\left(\mathcal{E}_{v}\right)
$$

where: $\Sigma$ is the 2-homology class, and $H_{\Sigma}+P . D .[\Sigma]$ is the equivariant version of its Poincare dual, $H_{\Sigma}$ is a function on $X$, linear in $p_{i}$, and $H_{\Sigma}^{(v)}$ its value at the fixed point $v$. Finally, $\mathcal{E}_{v}$ is the localized universal sheaf, i.e. $\iota^{v *} \mathcal{E}$, where $\iota^{v}: \mathfrak{M} \times\{v\} \hookrightarrow \mathfrak{M} \times X$ is the inclusion. 
In the calculation of the bosonic and fermionic determinants one uses the equivariant version of Atiyah-Singer index theorem, which yields the character of the tangent space to the moduli space of torsion free sheaves:

$$
C h T \mathfrak{M}=\int_{X} C h \mathcal{E} C h \mathcal{E}^{*} T d_{X}=\sum_{v} \frac{C h \mathcal{E}_{v} C h \mathcal{E}_{v}^{*}}{\left(1-e^{w_{v 1}}\right)\left(1-e^{w_{v 2}}\right)}
$$

\subsubsection{Examples}

(1) Let us consider $X=\mathbb{C P}^{d}$, which corresponds to $M=1$. In this case $\Delta_{X}$ is just a simplex,

$$
\sum_{i} p_{i}=r
$$

(if $r>0$, of course). The fixed points $v$ are in one-to-one correspondence with the vertices of the simplex, i.e. we can set $v=1, \ldots M+1$, $\vec{p}_{v}=\left(0, \ldots, r, \ldots, 0^{0}\right)$.

(2) The next example is non-compact. $X$ is the total space of the vector bundle $\mathcal{O}\left(q_{1}\right) \oplus \mathcal{O}\left(q_{d-1}\right)$ over $\mathbb{P}^{1}$. It corresponds to $M=1$, the vector of charges:

$$
\vec{Q}=\left(q_{1}, q_{2}, \ldots, q_{d-1}, 1,1\right)^{t}
$$

There are two fixed points $v$ - they belong to the zero section of the vector bundle, and are the North and South poles of the sphere.

\section{MASTER FORMULA}

We are now almost ready to state our main formula.

We first fix the vacuum expectation value of the Higgs field $\Phi$,

$$
\langle\Phi\rangle=\vec{a} \equiv \operatorname{diag}\left(a_{1}, \ldots, a_{N}\right)
$$

In the case of compact $X$ we should take the integral over $a$, to take into account the tunneling between different vacua.

The second cohomology group of $X$ is isomorphic to $\mathbb{Z}^{d}$, generically. The $U(N)$ gauge bundle is reduced to the $U(1)^{N}$ bundle, in the presence of the Higgs vev. We should sum over all equivalence classes of the $U(1)^{N}$ bundles. They are classified by $d$ vectors $\vec{k}_{a}=\left(k_{a, l}\right), a=1, \ldots, d$. In the $S U(N) / \mathbb{Z}_{N}$ gauge theory one fixes the traces: $\left\{\vec{k}_{a}\right\}=w_{a}=\sum_{l=1}^{N} k_{a, l}$. Actually, in the absence of the charged matter, everything depends only on $w_{a} \operatorname{modN}$. The partition function of the $\mathcal{N}=2$ theory in $\Omega$-background on the toric 
manifold $X$, in the presence of 2-observables,

$$
\left\langle\exp \sum_{\vec{n}} \int \mathbf{t}_{\vec{n}} \mathcal{O}_{\mathcal{O}_{\vec{n}}}^{(2)}\right\rangle, \quad \mathbf{t}_{\vec{n}} \in H_{c}^{2}(X, \mathbb{Z})
$$

is given by:

$$
Z_{\vec{w}}\left(\vec{a}, \vec{\varepsilon} ; \tau_{\vec{n}}, \mathbf{t}_{\vec{n}}\right)=\sum_{\vec{k}_{a} \in \mathbb{Z}^{N},\left\{\vec{k}_{a}\right\}=w_{a}} \prod_{v} Z\left(\vec{a}+\sum_{a} k_{a} \phi_{a}^{(v)}(\varepsilon), w_{v 1}, w_{v 2} ; \tau_{\vec{n}}+\mathbf{t}_{\vec{n}} H_{v}\right)
$$

It follows more or less directly from the Eq. (66)

\subsection{Non-equivariant limit}

In the case of compact $X$ the partition function (68) has a finite nonequivariant limit, i.e. the limit where $\vec{\varepsilon} \rightarrow 0$. Indeed, the singular terms in the logarithm of partition function vanish thanks to the identity:

$$
\sum_{v} \frac{1}{w_{v 1} w_{v 2}}=\int_{X} 1=0, \quad \sum_{v} \frac{\phi_{a}^{(v}(\varepsilon),\left(w_{v 1}+w_{v 2}\right)}{w_{v 1} w_{v 2}}=\int_{X} c_{1}\left(L_{a}\right), c_{1}(X)=0
$$

and the finite part reduces to:

$$
\begin{array}{r}
\mathcal{Z}_{\vec{w}}\left(\vec{a} ; \tau_{\vec{n}}, \mathbf{t}_{\vec{n}}\right)=\sum_{\vec{k}_{a} \in \mathbb{Z}^{N},\left\{\vec{k}_{a}\right\}=w_{a}} \exp \int_{X} \mathcal{F}_{0}\left(\vec{a}+\sum_{a} k_{a} c_{1}\left(L_{a}\right)\right)+ \\
+c_{1}(X) H\left(\vec{a}+\sum_{a} k_{a} c_{1}\left(L_{a}\right)\right)+\chi(X) \mathcal{F}_{1}(\vec{a})+\sigma(X) \mathcal{G}_{1}(\vec{a})
\end{array}
$$

IF we now integrate over $\vec{a}$ then the resulting function of $\vec{T}, \vec{t}$ (for $\vec{T}=$ $(\log \Lambda, 0,0, \ldots))$ is, in fact, the generating function of Donaldson invariants of $X$, for some specific choice of the metric on $X$. This choice is correlated with the choice of contour for the $\vec{a}$ integral.

\subsection{Non-holomorphic speculations}

Looking at the non-equivariant limit of the partition function we recognize the holomorphic approach formulae of Ref. [42], see also Ref. [28] . There, the low-energy effective theory of $\mathcal{N}=2$ super Yang-Mills theory was exploited in order to write down a contribution of the Coulomb branch to the Donaldson invariants of manifolds with $b_{2}^{+}=1$ (all toric manifolds are have this property). More systematic approach, taking into account full multiplet of 
$\mathcal{N}=2$ susy leads to the non-holomorphic integrand, see Ref. [42,50], whose integral can be converted to the contour integral of a holomorphic form, similar to what we have above. We hope to infer from this the non-holomorphic modular completion of our master formula.

\subsection{Relation to the results of Nakajima-Yoshioka}

This section is devoted to the simplest non-trivial example of the application of the formula (68): $X=\hat{\mathbb{C}}^{2}$, the blowup of a point on $\mathbb{C}^{2}$. It is a toric manifold, $M=1, d=2$. In this case the formula (68) reduces to:

$$
\begin{aligned}
& Z_{X, w}\left(\vec{a}, \epsilon_{1}, \epsilon_{2}, \epsilon_{3} ; \tau_{\vec{n}}, \mathbf{t}_{\vec{n}}\right)= \\
& \sum_{\vec{k} \in \mathbb{Z}^{N},\{\vec{k}\}=w} Z\left(\vec{a}+\vec{k} \epsilon_{1}, \epsilon_{1}+\epsilon_{3}, \epsilon_{2}-\epsilon_{1} ; \tau_{\vec{n}}+\mathbf{t}_{\vec{n}}\left(\epsilon_{1}+\epsilon_{3}\right)\right) \times \\
& Z\left(\vec{a}+\vec{k} \epsilon_{2}, \epsilon_{1}-\epsilon_{2}, \epsilon_{2}+\epsilon_{3} ; \tau_{\vec{n}}+\mathbf{t}_{\vec{n}}\left(\epsilon_{2}+\epsilon_{3}\right)\right)
\end{aligned}
$$

which, for $\epsilon_{3}=0$ coincides with the result of Ref. [53].

\section{Acknowledgments}

I would like to thank the organizers of the Congress for the beautiful conference, and for inviting me to give a lecture.

I also wish to thank A. Losev and A. Okounkov for useful discussions. Research was partially supported by 01-01-00549 grant from RFFI.

\section{References}

1. M. Aganagic, M. Mariño, C. Vafa, hep-th/0206164

2. O. Aharony, M. Berkooz, N. Seiberg, hep-th/9712117

3. O. Aharony, M. Berkooz, S. Kachru, N. Seiberg, E. Silverstein, hep-th/9707079

4. I. Antoniadis, E. Gava, K. Narain, T. Taylor, Nucl. Phys. B413 (1994) 162

5. M. Atiyah, V. Drinfeld, N. Hitchin, Yu. Manin, Phys. Lett. 65A (1978) 185

6. M. Atiyah, G. Segal, Ann. of Math. 87 (1968) 531

7. V. Balasubramanian, P. Kraus, A. Lawrence, hep-th/9805171

8. L. Baulieu, A. Losev, N. Nekrasov, hep-th/9707174

9. M. Bershadsky, S. Cecotti, H. Ooguri, C. Vafa, "Kodaira-Sprencer theory of gravity and exact results for quantum string amplitudes", Comm. Math. Phys. 165, 311-428 (1994), hep-th/9309140

10. H. Braden, N. Nekrasov, hep-th/9912019

11. U. Bruzzo, F. Fucito, J.F. Morales, A. Tanzini, hep-th/0211108

D.Bellisai, F.Fucito, A.Tanzini, G.Travaglini, hep-th/0002110, hep-th/0003272, hepth/0008225

R. Flume, R. Poghossian, hep-th/0208176 
R. Flume, R. Poghossian, H. Storch, hep-th/0110240, hep-th/0112211

N. Dorey, T.J. Hollowood, V. Khoze, M. Mattis, hep-th/0206063, and references therein 12. F. Cachazo, M. Douglas, N. Seiberg, E. Witten, hep-th $/ 0211170$

13. S. Cecotti, L. Girardello, Phys. Lett. 110B (1982) 39

14. A. Connes, "Noncommutative geometry", Academic Press (1994)

15. A. Connes, M. Douglas, A. Schwarz, JHEP 9802(1998) 003

16. E. Corrigan, P. Goddard, "Construction of instanton and monopole solutions and reciprocity", Ann. Phys. 154 (1984) 253

17. D.-E. Diaconescu, M. Douglas, J. Gomis, hep-th/9712230

18. R. Dijkgraaf, hep-th/9609022

19. R. Dijkgraaf, C. Vafa, hep-th/0206255, hep-th/0207106, hep-th/0208048

R. Dijkgraaf, S. Gukov, V. Kazakov, C. Vafa, hep-th/0210238

R. Dijkgraaf, M. Grisaru, C. Lam, C. Vafa, D. Zanon, hep-th/0211017

M. Aganagic, M. Marino, A. Klemm, C. Vafa, hep-th/0211098

R. Dijkgraaf, A. Neitzke, C. Vafa, hep-th/0211194

20. M. Douglas, hep-th/9311130, hep-th/9303159

21. M. Douglas, V. Kazakov, hep-th/9305047

22. J. J. Duistermaat, G.J. Heckman, Invent. Math. 69 (1982) 259

M. Atiyah, R. Bott, Topology 23 No 1 (1984) 1

M. Atiyah, R. Bott, Phil. Trans. Roy. Soc. London A 308 (1982), 524-615

E. Witten, hep-th/9204083

S. Cordes, G. Moore, S. Rangoolam, hep-th/9411210

R. Bott, J. Diff. Geom. 4 (1967) 311

G. Ellingsrud, S.A.Stromme, Invent. Math. 87 (1987) 343-352

L. Göttche, Math. A.. 286 (1990) 193-207

23. O. Ganor, hep-th/9607092, hep-th/9608108

24. A. Gorsky, A. Marshakov, A. Mironov, A. Morozov, Nucl. Phys. B527 (1998) 690-716, hep-th/9802007

25. R. Gopakumar, C.Vafa, hep-th/9809187, hep-th/9812127

26. A.Gorsky, I.Krichever, A.Marshakov, A.Mironov and A.Morozov, Phys. Lett. B355 (1995) 466; hep-th/9505035.

27. A. Gerasimov, A. Levin, A. Marshakov, Nucl. Phys. B360 (1991) 537

A. Bilal, I. Kogan, V. Fock, Nucl. Phys. B359 (1991) 635

28. L. Göttsche, D.Zagier, alg-geom/9612020

29. D. Gross, hep-th/9212149

D. Gross, W. Taylor, hep-th/9301068, hep-th/9303046

30. A. Iqbal, hep-th/0212279

31. A. Iqbal, N. Nekrasov, A. Okounkov, C. Vafa, "Quantum foam and topological strings", ITEP-TH-60/03 ,IHES/P/03/65, hep-th/0312022

32. S. Kachru, A. Klemm, W. Lerche, P. Mayr, C. Vafa, hep-th/9508155

S. Kachru, C. Vafa, hep-th/9505105

33. S. Kharchev, hep-th/9810091

34. A. Klemm, W. Lerche, P. Mayr, C. Vafa, N. Warner, hep-th/9604034

35. A. Klemm, W. Lerche, S. Theisen, S. Yankielowicz, hep-th/9411048

P. Argyres, A. Faraggi, hep-th/9411057

A. Hanany, Y. Oz, hep-th/9505074

36. I. Krichever, hep-th $/ 9205110$

37. M. Kontsevich, hep-th/9405035

38. A. Lawrence, N. Nekrasov, C. Vafa, hep-th/9803015

39. A. Losev, A. Marshakov, N. Nekrasov, "Small Instantons, Little Strings and Free 
Fermions", ITEP-TH-18/03, MPIM-2003-26, FIAN/TD-05/03, IHES-P/03/09, hepth/0302191

40. A. Losev, G. Moore, S. Shatashvili, hep-th/9707250

N. Seiberg, hep-th/9705221

41. A. Losev, G. Moore, N. Nekrasov, S. Shatashvili, hep-th/9509151

42. A. Losev, N. Nekrasov, S. Shatashvili, hep-th/9711108, hep-th/9801061

43. A. Losev, N. Nekrasov, S. Shatashvili, hep-th/9908204, hep-th/9911099

44. I. Macdonald, "Symmetric functions and Hall polynomials", Oxford University Press, 1998

45. J. Maldacena, hep-th/9711200

S. Gubser, I. Klebanov, A. Polyakov, hep-th/9802109

E. Witten, hep-th/9802150

46. A. Marshakov, "Seiberg-Witten Theory and Integrable Systems," World Scientific, Singapore (1999)

"Integrability: The Seiberg-Witten and Whitham Equations", Eds. H. Braden and I. Krichever, Gordon and Breach (2000).

47. D. Maulik, N. Nekrasov, A. Okounkov, R. Pandharipande, "Gromov-Witten theory and Donaldson-Thomas theory", ITEP-TH-61/03, IHES/M/03/67, math.AG/0312059

48. G. Moore, N. Nekrasov, S. Shatashvili, "D-particle bound states and generalized instantons" Comm. Math. Phys. 209, 77-95 (2000), hep-th/9803265

49. G. Moore, N. Nekrasov, S. Shatashvili, hep-th/9712241, hep-th/9803265

50. G. Moore, E. Witten, hep-th/9709193

51. J. A. Minahan, D. Nemeschansky, C. Vafa, N.P. Warner, hep-th/9802168

T. Eguchi, K. Sakai, hep-th/0203025, hep-th/0211213

52. H. Nakajima, "Lectures on Hilbert Schemes of Points on Surfaces" AMS University Lecture Series, 1999, ISBN 0-8218-1956-9.

53. H. Nakajima, K. Yoshioka, math.AG/0306198, math.AG/0311058

54. N. Nekrasov, hep-th/0010017, hep-th/0203109

55. N. Nekrasov, A. Okounkov, "Equivariant vertex", in preparation

56. N. Nekrasov, A. Okounkov, "Seiberg-Witten theory and random partitions", ITEPTH-36/03, IHES-P/03/43, hep-th/0306238

57. N. Nekrasov, "Seiberg-Witten prepotential from instanton counting", hep-th/0206161

58. N. Nekrasov, hep-th/9609219

A. Lawrence, N. Nekrasov, hep-th/9706025

59. N. Nekrasov, A. S. Schwarz, hep-th/9802068

60. V. Novikov, M. Shifman, A. Vainshtein, V. Zakharov, Phys. Lett. 217B (1989) 103

61. A. Okounkov, R. Pandharipande, math.AG/0207233, math.AG/0204305

62. A. Okounkov, N. Reshetikhin, "Correlation function of Schur process with application to local geometry of a random 3-dimensional Young diagram", math.CO/0107056

63. A. Okounkov, N. Reshetikhin, C. Vafa, "Quantum Calabi-Yau and Classical Crystals", hep-th/0309208

64. A. Polyakov, hep-th/9711002, hep-th/9809057

65. N. Seiberg, E. Witten, hep-th/9407087, hep-th/9408099

66. N. Seiberg, hep-th/9408013

67. S. Sethi, M. Stern, "D-brane bound state redux", Comm. Math. Phys. 194, 675-705 (1998), hep-th/9705046

68. A. Smilga, Yad.Fiz. 43 (1986), 215-218

69. C. Vafa, hep-th/0008142

70. S. Katz, A. Klemm, C. Vafa, hep-th/9609239

71. A. Klemm, E. Zaslow, hep-th/9906046 
72. I. Klebanov, N. Nekrasov, hep-th/9911096

J. Polchinski, hep-th/0011193

73. A. Marshakov, A. Mironov, hep-th/9711156

H. Braden, A. Marshakov, A. Mironov, A. Morozov, hep-th/9812078, hep-th/9902205

T. Eguchi, H. Kanno, hep-th/0005008

H. Braden, A. Marshakov, hep-th/0009060

H. Braden, A. Gorsky, A. Odesskii, V. Roubtsov, hep-th/01111066

C.Csaki, J.Erlich, V.V.Khoze, E.Poppitz, Y.Shadmi, Y.Shirman, hep-th/0110188

T. Hollowood, hep-th/0302165

74. N. Seiberg, hep-th/9608111

75. N. Seiberg, E. Witten, hep-th/9908142, JHEP 9909(1999) 032

76. K. Ueno, K. Takasaki, Adv. Studies in Pure Math. 4 (1984) 1

77. A. M. Vershik, "Hook formulae and related identities", Zapiski sem. LOMI, 172 (1989), 3-20 (in Russian)

S. V. Kerov, A. M. Vershik, "Asymptotics of the Plancherel measure of the symmetric group and the limiting shape of the Young diagrams", DAN SSSR, 233 (1977), 10241027 (in Russian)

S. V. Kerov, "Random Young tableaux", Teor. veroyat. i ee primeneniya, 3 (1986), 627-628 (in Russian)

78. E. Witten, Comm. Math. Phys. 117 (1988) 353

79. E. Witten, hep-th/9403195

80. E. Witten, hep-th/9503124

81. E. Witten, hep-th/9510153

82. E. Witten, hep-th/9703166 